\title{
Recent progress on the tidal deformability of spinning compact objects
}

\author{
Paolo Pani ${ }^{1,2, *}$, Leonardo Gualtieri ${ }^{1}$, Andrea Maselli $^{3}$, Valeria Ferrari ${ }^{1}$ \\ ${ }^{1}$ Dipartimento di Fisica, "Sapienza" Università di Roma \&S Sezione INFN Roma1, Piazzale \\ Aldo Moro 5, 00185, Roma, Italy. \\ ${ }^{2}$ CENTRA, Departamento de Física, Instituto Superior Técnico, Universidade de Lisboa, \\ Avenida Rovisco Pais 1, 1049 Lisboa, Portugal. \\ ${ }^{3}$ Theoretical Astrophysics, Eberhard Karls University of Tuebingen, Tuebingen 72076, Germany. \\ *E-mail: paolo.pani@roma1.infn.it
}

\begin{abstract}
We give a short review on recent progress in the theory of tidal deformability of a slowly spinning compact object. A rotating object immersed in a quadrupolar, electric tidal field can acquire some induced mass, spin, quadrupole, octupole and hexadecapole moments to second order in the spin. Angular momentum introduces couplings between electric and magnetic distortions and new classes of spin-induced, tidal Love numbers emerge. All tidal Love numbers of a Kerr black hole were proved to be exactly zero to first order in the spin and also to second order in the spin, at least in the axisymmetric case. The tidal Love numbers of a neutron star depend strongly on the equation of state. Spintidal couplings deteriorate some approximate universal relations that exist for neutron stars in the static case. For a binary system close to the merger, various components of the tidal field become relevant. Preliminary results suggest that spin-tidal couplings can introduce important corrections to the gravitational waveforms of spinning neutron-star binaries approaching the merger.
\end{abstract}

\section{Introduction}

In the last few years there has been considerable progress in understanding the tidal deformations of a spinning compact object. The motivation for these studies is twofold. On the one hand a deeper theoretical understanding of the tidal deformability within general relativity is highly desirable, because the nonlinearities of the theory introduce some subtleties in the definition of the tidal Love numbers ${ }^{1,2}$ for relativistic compact objects ${ }^{3,4}$. On the other hand, the first direct detection of gravitational waves $(\mathrm{GWs})$ by aLIGO ${ }^{5}$, the prospects of measuring the tidal Love numbers through $\mathrm{GW}$ detections of compact binaries ${ }^{6-18}$, and the related possibility of constraining the equation of state (EoS) of neutron stars (NSs) through GW astronomy, make it urgent to include spin-tidal effects in the gravitational waveforms. In this work we briefly review some recent results on the deformability and tidal Love numbers of spinning black holes (BHs) and NSs.

\section{Selection rules for the tidal deformations of a spinning object}

Before reviewing the main results, let us discuss some selection rules that govern the deformations of a spinning object immersed in a tidal field. As a result of the external perturbation, the mass and current multipole moments ${ }^{19-21}$ (denoted by $M_{\ell}$ and $S_{\ell}$, respectively) of the compact object will be deformed. In linear 
perturbation theory, for a nonspinning object one can define the standard (electric and magnetic) tidal Love numbers ${ }^{7,22,23}$ as

$$
\lambda_{E}^{(\ell)} \equiv \frac{\partial M_{\ell}}{\partial \mathcal{E}_{m}^{(\ell)}}, \quad \lambda_{M}^{(\ell)} \equiv \frac{\partial S_{\ell}}{\partial \mathcal{B}_{m}^{(\ell)}},
$$

where $\mathcal{E}_{m}^{(\ell)}$ and $\mathcal{B}_{m}^{(\ell)}$ denote ${ }^{24}$ the amplitude of the electric and magnetic components of the external tidal field with harmonic indices $(\ell, m)$, where $m$ is the azimuthal number ${ }^{\mathrm{a}}$. When the object is spherically symmetric, $m$ is degenerate and parity and the angular momentum number $\ell$ are conserved: an electric (i.e. even parity) tidal field with harmonic index $\ell$ can only deform the mass multipole moment of order $\ell$, whereas a magnetic (i.e. odd parity) tidal field with harmonic index $\ell$ can only deform the current multipole moment of order $\ell$. When the central object is spinning such degeneracy is broken and there exist some selection rules ${ }^{24,25}$ which allow to define a more generic set of tidal Love numbers,

$$
\begin{array}{ll}
\lambda_{E,-}^{\left(\ell \ell^{\prime} m\right)} \equiv \frac{\partial M_{\ell}}{\partial \mathcal{B}_{m}^{\left(\ell^{\prime}\right)}}, & \lambda_{M,-}^{\left(\ell \ell^{\prime} m\right)} \equiv \frac{\partial S_{\ell}}{\partial \mathcal{E}_{m}^{\left(\ell^{\prime}\right)}}, \\
\lambda_{E,+}^{\left(\ell \ell^{\prime} m\right)} \equiv \frac{\partial M_{\ell}}{\partial \mathcal{E}_{m}^{\left(\ell^{\prime}\right)}}, & \lambda_{M,+}^{\left(\ell \ell^{\prime} m\right)} \equiv \frac{\partial S_{\ell}}{\partial \mathcal{B}_{m}^{\left(\ell^{\prime}\right)}},
\end{array}
$$

which enjoy various interesting properties ${ }^{24}$ :

(1) At zeroth order in the spin, the Love numbers in Eq. (2) vanish, whereas the Love numbers in Eq. (3) are different from zero only when $\ell^{\prime}=\ell$ and reduce to those defined in Eq. (1). The azimuthal number $m$ is degenerate in this case.

(2) At first order in the spin, the Love numbers in Eq. (2) are different from zero only when $\ell^{\prime}=\ell \pm 1$, whereas the Love numbers in Eq. (3) are different from zero only when $\ell^{\prime}=\ell$ and in the nonaxisymmetric case, $m \neq 0$.

(3) At second order in the spin, the Love numbers in Eq. (2) acquire new corrections only in the nonaxisymmetric case, whereas the Love numbers in Eq. (3) are different from zero both when $\ell^{\prime}=\ell$ and when $\ell^{\prime}=\ell \pm 2$ for any value of $m$. The terms $\lambda_{E,+}^{(\ell \ell m)}$ and $\lambda_{M,+}^{(\ell \ell m)}$ include $\mathcal{O}\left(\chi^{2}\right)^{\mathrm{b}}$ corrections to the static Love numbers in Eq. (1).

\section{Tidal deformations of spinning $\mathrm{BHs}$}

The geometry of a tidally-deformed, spinning BH was obtained by Yunes \& Gonzalez through an integration of the Teukolsky equations ${ }^{26}$, whereas more recently the intrinsic geometry of a spinning BH distorted by a small compact companion has been studied in the extreme-mass ratio limit by O'Sullivan and Hughes ${ }^{27,28}$. These papers considered arbitrary values of the BH spin parameter but are limited

\footnotetext{
${ }^{a}$ We adopt the same decomposition of the tidal field as Binnington \& Poisson ${ }^{22}$.

${ }^{\mathrm{b}}$ Henceforth $\chi:=J / M^{2} \ll 1$, where $M$ and $J$ are the object's mass and angular momentum.
} 
to numerical (or semi-analytical) analysis. An analytical study of the tidal deformations of a spinning $\mathrm{BH}$ was initiated by Poisson ${ }^{25}$, who computed the tidal deformations of a Kerr $\mathrm{BH}$ immersed in a generic quadrupolar tidal field to first order in the spin. He constructed a set of coordinates which are regular on the $\mathrm{BH}$ horizon, decomposed the external tidal field in terms of irreducible potentials, and extracted the tidal moments through a matching to the post-Newtonian metric of a binary system. As a result of this procedure, the metric of a tidally deformed Kerr $\mathrm{BH}$ to first order in the spin is given explicitly in closed form ${ }^{25}$.

For axisymmetric tidal fields, such results have been extended to include secondorder spin corrections for a quadrupolar electric tidal field ${ }^{29}$, and to include both electric and magnetic tidal fields with $\ell=3,4$ to first order in the $\operatorname{spin}^{24}$.

The main result of these studies is the analytical proof that the rotational tidal Love numbers of a Kerr BH are precisely zero ${ }^{24,29,30}$, as in the Schwarzschild case ${ }^{4,22,31,32}$. This result is a direct consequence of the regularity of the tidal perturbation on the event horizon, and has important implications. Indeed, the multipole moments of any stationary $\mathrm{BH}$ in isolation can be written as ${ }^{20}$

$$
M_{\ell}+i S_{\ell}=M^{\ell+1}(i \chi)^{\ell},
$$

and therefore all moments with $\ell \geq 2$ can be written in terms of $M_{0}=M$ and $S_{1}=J$ through the above relation. Any independent measurement of three multipole moments (e.g. the mass, the spin and the mass quadrupole $M_{2}$ ) is a null-test of the Kerr metric and, in turn, it might provide a genuine strong-gravity confirmation of general relativity ${ }^{33-36}$. The results mentioned above show that the no-hair relations (4) hold also for a slowly rotating BH immersed in a weak tidal field and can therefore be interpreted as a generalization of the no-hair theorems for stationary, tidally-deformed spinning BHs.

In light of these results, it is natural to conjecture that the Love numbers of a Kerr BH are zero to any order in the spin. Verifying this important conjecture requires an extension of current analyses (at least) to second order in the spin and in the nonaxisymmetric case.

\section{Tidal deformations of spinning NSs}

The tidal Love numbers of a NS depend on the object's internal structure, namely on the NS mass and on the EoS of the matter composing the star. Measuring the NS deformability through GW detections would help to constrain the behavior of matter at ultranuclear density ${ }^{18,37-40}$. This has motivated a vast literature on the tidal deformability of nonspinning NSs. On the other hand, spin effects have been considered only recently within a perturbative scheme. Landry \& Poisson ${ }^{30}$ computed the external metric of a spinning material object immersed in a generic quadrupolar (both electric and magnetic) tidal field to first order in the spin, whereas Pani et al. ${ }^{24}$ considered an axisymmetric tidal field with $\ell=2,3,4$ and computed the rotational Love numbers (2)-(3) to first order in the spin for various tabulated EoS. 
Two of the main results of the latter analysis are:

(1) For a binary system close to the merger, various components of the tidal field become relevant. In this case it was found that an octupolar magnetic tidal field can significantly modify the mass quadrupole moment of a NS. Assuming a spin parameter $\chi \approx 0.05$ and an orbital distance $r_{0} \approx 5 R$, deformations can differ by $\gtrsim 10 \%$ relative to the static case. These results suggest that spin-tidal couplings can introduce important corrections to the gravitational waveforms of a spinning NS binary system.

(2) Some of the rotational Love numbers deviate from the approximately universal relations that hold for nonrotational Love numbers ${ }^{41-43}$ by an amount as large as $200 \%$ in the electric-led case and as large as $50 \%$ in the magnetic-led case. The approximate universality of the induced mass quadrupole moment deteriorates from $1 \%$ in the static case to roughly $6 \%$ when $\chi \approx 0.05$ and $r_{0} \approx 5 R$.

Finally, very recently Landry \& Poisson ${ }^{44,45}$ have studied the gravitomagnetic response of an irrotational self-gravitating fluid to an external tidal field to first order in the angular momentum. Remarkably, they found that in such condition the irrotational fluid undergoes a dynamical (i.e. time-dependent) response on the time scale of the rotation period, whereas the exterior geometry remains stationary. This result also shows the existence of a novel family of magnetic-led Love numbers, which have been studied so far only in the nonspinning case ${ }^{44}$.

\section{Open issues}

We wish to conclude with a list of open problems related to the theory and phenomenology of the tidal deformability of compact objects:

(1) The computation of the tidal Love numbers relies on the separation - in the perturbed metric - of the external tidal field from the linear response of the object. Even at the linearized level this procedure presents some subtleties ${ }^{3,4,29}$ and a more rigorous analysis would be highly desirable, even in the static case. This would likely require a 5th-order post-Newtonian expansion of the field equations for a binary system with comparable masses.

(2) Are the tidal Love numbers of a Kerr BH zero to any order in the spin?

(3) Are the tidal Love numbers of a $\mathrm{BH}$ zero also in gravitational theories other than general relativity?

(4) Spin-tidal interactions seem to have a nonnegligible impact on the NS deformability. A more rigorous analysis is needed to quantify their impact on the gravitational waveforms of a spinning NS binary system. A promising approach to incorporate such effects in the relativistic two-body dynamics is by extending effective point-particle techniques ${ }^{46}$ to include spin-tidal couplings. 


\section{Acknowledgments}

This work was supported by FCT-Portugal through Project No. IF/00293/2013 and by the COST Action MP1304 "NewCompStar".

\section{References}

1. C. Murray and S. Dermott, Solar System Dynamics (Cambridge University Press, 2000).

2. E. Poisson and C. Will, Gravity: Newtonian, Post-Newtonian, Relativistic 2014.

3. T. Damour and O. M. Lecian, On the gravitational polarizability of black holes, Phys.Rev. D80, p. 044017 (2009).

4. B. Kol and M. Smolkin, Black hole stereotyping: Induced gravito-static polarization, JHEP 1202, p. 010 (2012).

5. B. . Abbott et al., Observation of Gravitational Waves from a Binary Black Hole Merger, Phys. Rev. Lett. 116, p. 061102 (2016).

6. E. E. Flanagan and T. Hinderer, Constraining neutron star tidal Love numbers with gravitational wave detectors, Phys.Rev. D77, p. 021502 (2008).

7. T. Hinderer, Tidal Love numbers of neutron stars, Astrophys.J. 677, 1216 (2008).

8. L. Baiotti, T. Damour, B. Giacomazzo, A. Nagar and L. Rezzolla, Analytic modelling of tidal effects in the relativistic inspiral of binary neutron stars, Phys.Rev.Lett. 105, p. 261101 (2010).

9. L. Baiotti, T. Damour, B. Giacomazzo, A. Nagar and L. Rezzolla, Accurate numerical simulations of inspiralling binary neutron stars and their comparison with effectiveone-body analytical models, Phys.Rev. D84, p. 024017 (2011).

10. J. Vines, E. E. Flanagan and T. Hinderer, Post-1-Newtonian tidal effects in the gravitational waveform from binary inspirals, Phys.Rev. D83, p. 084051 (2011).

11. F. Pannarale, L. Rezzolla, F. Ohme and J. S. Read, Will black hole-neutron star binary inspirals tell us about the neutron star equation of state?, Phys.Rev. D84, p. 104017 (2011).

12. J. E. Vines and E. E. Flanagan, Post-1-Newtonian quadrupole tidal interactions in binary systems, Phys.Rev. D88, p. 024046 (2013).

13. B. D. Lackey, K. Kyutoku, M. Shibata, P. R. Brady and J. L. Friedman, Extracting equation of state parameters from black hole-neutron star mergers. I. Nonspinning black holes, Phys.Rev. D85, p. 044061 (2012).

14. B. D. Lackey, K. Kyutoku, M. Shibata, P. R. Brady and J. L. Friedman, Extracting equation of state parameters from black hole-neutron star mergers: aligned-spin black holes and a preliminary waveform model, Phys.Rev. D89, p. 043009 (2014).

15. M. Favata, Systematic parameter errors in inspiraling neutron star binaries, Phys.Rev.Lett. 112, p. 101101 (2014).

16. K. Yagi and N. Yunes, Love can be Tough to Measure, Phys.Rev. D89, p. 021303 (2014).

17. A. Maselli, V. Cardoso, V. Ferrari, L. Gualtieri and P. Pani, Equation-of-stateindependent relations in neutron stars, Phys.Rev. D88, p. 023007 (2013).

18. A. Maselli, L. Gualtieri and V. Ferrari, Constraining the equation of state of nuclear matter with gravitational wave observations: Tidal deformability and tidal disruption, Phys.Rev. D88, p. 104040 (2013).

19. R. P. Geroch, Multipole moments. II. Curved space, J.Math.Phys. 11, 2580 (1970).

20. R. Hansen, Multipole moments of stationary space-times, J.Math.Phys. 15, 46 (1974). 
21. K. Thorne, Multipole Expansions of Gravitational Radiation, Rev.Mod.Phys. 52, 299 (1980).

22. T. Binnington and E. Poisson, Relativistic theory of tidal Love numbers, Phys.Rev. D80, p. 084018 (2009).

23. T. Damour and A. Nagar, Relativistic tidal properties of neutron stars, Phys.Rev. D80, p. 084035 (2009).

24. P. Pani, L. Gualtieri and V. Ferrari, Tidal Love numbers of a slowly spinning neutron star, Phys. Rev. D92, p. 124003 (2015).

25. E. Poisson, Tidal deformation of a slowly rotating black hole, Phys. Rev. D 91, p. 044004 (Feb 2015).

26. N. Yunes and J. Gonzalez, Metric of a tidally perturbed spinning black hole, Phys.Rev. D73, p. 024010 (2006).

27. S. O'Sullivan and S. A. Hughes, Strong-field tidal distortions of rotating black holes: Formalism and results for circular, equatorial orbits, Phys.Rev. D90, p. 124039 (2014).

28. S. O'Sullivan and S. A. Hughes, Strong-field tidal distortions of rotating black holes: II. Horizon dynamics from eccentric and inclined orbits (2015).

29. P. Pani, L. Gualtieri, A. Maselli and V. Ferrari, Tidal deformations of a spinning compact object, Phys. Rev. D92, p. 024010 (2015).

30. P. Landry and E. Poisson, Tidal deformation of a slowly rotating material body. I. External metric, Phys.Rev. D91, p. 104018 (2015).

31. N. Güerlebeck, No-hair theorem for Black Holes in Astrophysical Environments (2015).

32. S. Chakrabarti, T. Delsate and J. Steinhoff, New perspectives on neutron star and black hole spectroscopy and dynamic tides (2013).

33. D. Psaltis, Probes and Tests of Strong-Field Gravity with Observations in the Electromagnetic Spectrum (2008).

34. J. R. Gair, M. Vallisneri, S. L. Larson and J. G. Baker, Testing General Relativity with Low-Frequency, Space-Based Gravitational-Wave Detectors, Living Rev.Rel. 16, p. 7 (2013).

35. N. Yunes and X. Siemens, Gravitational-Wave Tests of General Relativity with Ground-Based Detectors and Pulsar Timing-Arrays, Living Rev.Rel. 16, p. 9 (2013).

36. E. Berti et al., Testing General Relativity with Present and Future Astrophysical Observations, Class. Quant. Grav. 32, p. 243001 (2015).

37. J. Lattimer and M. Prakash, The physics of neutron stars, Science 304, 536 (2004).

38. T. Hinderer, B. D. Lackey, R. N. Lang and J. S. Read, Tidal deformability of neutron stars with realistic equations of state and their gravitational wave signatures in binary inspiral, Phys.Rev. D81, p. 123016 (2010).

39. S. Postnikov, M. Prakash and J. M. Lattimer, Tidal Love Numbers of Neutron and Self-Bound Quark Stars, Phys.Rev. D82, p. 024016 (2010).

40. T. Damour, A. Nagar and L. Villain, Measurability of the tidal polarizability of neutron stars in late-inspiral gravitational-wave signals, Phys.Rev. D85, p. 123007 (2012).

41. K. Yagi and N. Yunes, I-Love-Q, Science 341, 365 (2013).

42. K. Yagi and N. Yunes, I-Love-Q Relations in Neutron Stars and their Applications to Astrophysics, Gravitational Waves and Fundamental Physics, Phys.Rev. D88, p. 023009 (2013).

43. K. Yagi, Multipole Love Relations, Phys.Rev. D89, p. 043011 (2014).

44. P. Landry and E. Poisson, Gravitomagnetic response of an irrotational body to an applied tidal field, Phys. Rev. D91, p. 104026 (2015). 
45. P. Landry and E. Poisson, Dynamical response to a stationary tidal field, Phys. Rev. D92, p. 124041 (2015).

46. R. A. Porto, The Effective Field Theorist's Approach to Gravitational Dynamics (2016). 